\title{
Study of serum cystatin C levels in patients with obstructive sleep apnea
} Eman Elfeky ${ }^{\mathrm{a}}$, Ayman Abd El-Zaher ${ }^{\mathrm{a}}$, Amal Elbendary ${ }^{\mathrm{b}}$, Salwa Ganna ${ }^{\mathrm{a}}$

\begin{abstract}
Background Serum cystatin C is a sensitive indicator for early renal impairment in patients with obstructive sleep apnea (OSA). In patients with OSA without known chronic kidney disease (CKD), serum cystatin $C$ was found to be elevated, indicating latent renal disease, which may be caused by recurrent episodes of hypoxemia and reoxygenation that trigger inflammatory process.
\end{abstract}

Objective To evaluate serum levels of cystatin $C$ in otherwise healthy patients with OSA to predict the future risk of latent renal impairment in these patients.

Patients and methods A total of 30 patients were classified into two groups: group I included 10 healthy controls, and group II included 20 patients with OSA.

The following were done: full history taking, clinical examination, pulmonary function tests, subjective evaluation of daytime sleepiness using the Epworth Sleepiness Scale, and overnight polysomnography. Finally, serum cystatin $\mathrm{C}$ levels at the end of polysomnography and glomerular filtration rate was calculated using modification of diet in renal disease formula and CKDEpidemiology Collaboration cystatin equation.

Results In patients with OSA, the mean level of serum cystatin $\mathrm{C}$ was statistically significantly higher than that in controls. These higher levels were sex, age, and BMI independently. Moreover, serum levels of cystatin C in patients with OSA were significantly correlated with OSA

\section{Introduction}

Obstructive sleep apnea (OSA) is characterized by recurrent attacks of either partial or complete upper airway collapse, resulting in hypopnea or apnea during sleep [1]. Several studies reported that chronic kidney disease (CKD) prevalence increases in patients with OSA, which is a known risk factor for cardiovascular disease (CVD), even in mild OSA $[2,3]$. Cystatin $\mathrm{C}$, a protein with a low molecular weight, is an inhibitor of lysosomal cysteine protease. It is mainly used as a biomarker for kidney function, as cystatin $\mathrm{C}$ is filtered by glomeruli and then reabsorbed and catabolized in the proximal convoluted tubules, but it is not secreted by the tubules [4]. In contrast to serum creatinine, cystatin $\mathrm{C}$ is not influenced by either muscle mass, age, or sex. So, it is a more sensitive indicator of mild and early kidney impairment and is a faster and a better estimate of glomerular filtration rate (GFR) than serum creatinine [5]. This work aimed at evaluation of serum levels of cystatin $\mathrm{C}$ in otherwise healthy patients with OSA to predict the future risk of latent renal impairment in these patients. severity (apnea-hypopnea index) and degree of nocturnal hypoxia through positive correlation with total sleep time less than $90 \%$, total sleep time less than $80 \%$, and respiratory disturbance index and negative correlation with average $\mathrm{SPO}_{2} \%$, lowest $\mathrm{SPO}_{2} \%$, and estimated glomerular filtration rate by CKD-Epidemiology Collaboration cystatin equation.

Conclusions Higher serum levels of cystatin $\mathrm{C}$ were detected in patients with OSA without known comorbidities, which indicates that these patients are at a higher risk of developing CKD. Intermittent hypoxia seems to have the main role in the progression of this process.

Egypt J Bronchol 2019 13:563-569

(C) 2019 Egyptian Journal of Bronchology

Egyptian Journal of Bronchology 2019 13:563-569

Keywords: chronic kidney disease, chronic kidney disease cystatin C, obstructive sleep apnea,

${ }^{a}$ Chest Department, ${ }^{b}$ Clinical Pathology Department, Faculty of Medicine, Tanta University, Egypt

Correspondence to Salwa A Ganna, MD, Chest Department, Faculty of Medicine, Tanta University, 31527 Egypt. Tel: +20403337544; fax: +20403407734 e-mail: sganna76@yahoo.com

Received: 9 April 2019 Revised: 14 August 2019 Accepted: 16 August 2019 Published: 25 October 2019

\section{Patients and methods}

This prospective analytic observational cross sectional study was conducted in the Sleep Laboratory Unit in Chest Department, Tanta University Hospitals, between July 2017 and February 2018. A total of 101 patients were evaluated for OSA; 42 patients were hypertensive, 29 were diabetic, 21 patients were cardiac, and nine patients had CKD. So, these 71 patients were excluded from the study, and only 30 patients were included. The local ethics committee of our center has approved the research protocol, and written informed consents were obtained from all participants.

The 30 patients were classified into two groups: group I included 10 healthy controls, matched to patients with OSA by sex, age, and BMI. All control persons are apparently healthy individuals with no history of any

This is an open access journal, and articles are distributed under the terms of the Creative Commons Attribution-NonCommercial-ShareAlike 4.0 License, which allows others to remix, tweak, and build upon the work non-commercially, as long as appropriate credit is given and the new creations are licensed under the identical terms. 
diseases, had normal physical examinations, and had apnea-hypopnea index (AHI) less than 5 event $/ \mathrm{h}$. Group II included 20 patients with OSA as confirmed by AHI more than or equal to 5 event/h. Patients were diagnosed with OSA if their AHI ranges from 5 events/h to less than 15 event $/ h$ and presented at least one of the following: loud snoring, daytime sleeping, fatigue, and breathing interruption during sleep [6]. Patients with AHI more than or equal to 15 events/h were diagnosed with OSA regardless of whether they had any additional compliant [6]. OSA was considered mild if AHI ranges from 5 events/h to less than 15 events/h, moderate if AHI ranges from 15 events/h to less than 30 , and severe if AHI more than or equal to 30 events $/ \mathrm{h}$. If $\mathrm{AHI}$ less than 5 events $/ \mathrm{h}$, the person was taken as healthy control. Patients with central sleep apnea diagnosed by polysomnography (PSG), patients with systemic hypertension, diabetic patients, previously diagnosed patients with OSA, patients who started treatment with either CPAP or oral appliances, patients with cardiac or hepatic diseases, and patients with known renal diseases were excluded from this study.

All 30 persons were subjected to the following:

(1) History taking, including personal history; medical history; and subjective evaluation of daytime sleepiness using the Epworth Sleepiness Scale, which is a short questionnaire aiming at evaluation of the possibility of falling asleep in different situations [7].

(2) Physical examination, which included measurement of height; weight; neck, hip, and waist circumferences; and waist/hip circumference ratio. BMI was calculated using the following formula: $\mathrm{BMI}=$ weight $(\mathrm{kg}) /$ height $^{2}(\mathrm{~m})$.

(3) Arterial blood gas analysis.

(4) Plain chest $\mathrm{x}$-ray (posteroanterior view)

(5) ECG.

(6) Pulmonary function testing.

(7) Overnight PSG was conducted in the Sleep Laboratory Unit in Chest Department, Tanta University Hospitals, at night during sleep for at least $6 \mathrm{~h}$ (started at $10 \mathrm{pm}$ ). Participants were connected to $\mathrm{SOMNO}$ screen ${ }^{\mathrm{TM}}$ plus $\mathrm{PSG}^{+}$ (SOMNO Medics GmbH, Germany), which included the following channels: pressure and flow (cannula and thermistor), snore (cannula and/or microphone), thoracic effort, abdominal effort, pulse oximetry, ECG, periodic leg movement, electrooculogram, electroencephalogram (10 electrodes) to detect total sleep time (TST), and sleep efficiency. Respiratory event analysis included AHI and respiratory disturbance index (RDI). It identified different types of apneas (obstructive, central, or mixed) and calculated their absolute values and indices. It estimated respiratory effort related arousal index and absolute value. It measured the snoring and arousal index. Oxygen saturation analysis determined the baseline, average, and minimal oxygen saturation. It estimated TST less than 90\% (time of sleep with oxygen saturation below 90\%) and TST less than $80 \%$, as well as oxygen desaturation index which is the number of times per hour of sleep that the blood's oxygen level drops by $3 \%$ or more from baseline. Heart rate analysis measured maximal, minimal, and average heart rate. The PSG analysis was done automatically for all participants by DOMNO software and then revised manually. Sleep stage and respiratory event scoring was done according to the standard criteria of the American Academy of Sleep Medicine (AASM), version 2.3 [8].

(8) Laboratory investigations: fasting venous blood samples were collected under complete aseptic conditions the morning after the study. Samples were divided correspondingly for the following:

(a) Routine laboratory investigations: fasting blood glucose considering diabetic range starting from $126 \mathrm{mg} / \mathrm{dl}$ [9], triglyceride, total cholesterol, urea, creatinine, and $\mathrm{C}$ reactive protein.

(b) Cystatin $\mathrm{C}$ serum concentration: $2 \mathrm{ml}$ of the venous sample is used after centrifugation within $1 \mathrm{~h}$ of collection. Serum samples were stored at $-25^{\circ} \mathrm{C}$ for cystatin $\mathrm{C}$ analysis at the end of the study. Cystatin $\mathrm{C}$ was measured by using a particle-enhanced turbidimetric immunoassay.

(9) Estimated glomerular filtration rate (eGFR) was measured by modification of diet in renal disease (MDRD) formula [10] and the CKD-Epidemiology Collaboration (EPI) cystatin $\mathrm{C}$ equation [11] adjusted for age, sex, and race.

\section{Statistical analysis}

Expression of quantitative data as mean $\pm \mathrm{SD}$ was done. Data were tested for normal distribution using Kruskal-Wallis test and Mann-Whitney test to evaluate the significance of difference among both groups using SPSS, version 20 (Statistical Package for Social Sciences, version 20, IBM). A $P$ value less than 0.05 was considered to be statistically significant. In addition, Pearson correlations were performed between serum cystatin $C$ levels and different variables. 


\section{Results}

This study included 30 patients: 20 were diagnosed as have OSA by PSG (AHI $\geq 5$ event/h), and 10 apparent healthy control persons.

Demographic and anthropometric characteristics of both patients with OSA and controls are shown in Table 1. No statistically significant difference was detected between both groups regarding age, sex, percent of smokers, and BMI.

Statistical comparison between the two studied groups regarding different PSG parameters is shown in Table 2. There was no statistically significant difference between both groups regarding TST and baseline $\mathrm{SPO}_{2}$, whereas average $\mathrm{SPO}_{2} \%$ and minimal $\mathrm{SPO}_{2} \%$ were found to be statistically significant lower in OSA group than in control group, with $P$ value of 0.001 for all parameters. TST less than $90 \%$, TST less than $80 \%$, RDI, snoring index, and arousal index were found to be statistically significant higher in OSA group than in control group, with $P$ value of 0.001 for all parameters, except arousal index, with $P$ value of 0.028 .

Regarding classification of OSA severity regarding AHI (Fig. 1), OSA was mild in four patients, moderate in four patients, and severe in 12 patients.
AHI ranged from 7.0 to 99.3 event/h, with mean \pm SD of $48.94 \pm 29.90 \mathrm{event} / \mathrm{h}$.

Statistical comparison between the two studied groups regarding different laboratory investigation is shown in Table 3. No significant differences were detected between both groups regarding $\mathrm{ABG}$, fasting blood glucose, triglyceride, total cholesterol, blood urea, serum creatinine, and eGFR by MDRD formula. However, eGFR by CKD-EPI cystatin $\mathrm{C}$ equation was found to be statistically significant lower in OSA patients than control group. Moreover, serum cystatin C levels were statistically significant higher in OSA group than control group.

Receiver operating characteristic curve analysis (Table 4, Fig. 2) showed that cutoff value of eGFR by CKD-EPI equation in predicting renal injury was $60 \mathrm{ml} \mathrm{min} 1.73 \mathrm{~m}^{2}$, with a sensitivity of $95 \%$, specificity of $100 \%$, positive predictive value of $100 \%$, negative predictive value of $91 \%$, and accuracy of $97 \%$.

Statistical correlation between serum levels of cystatin C and some studied parameters in patients with OSA is shown in Table 5. The OSA group revealed that there was no statistically significant correlation between serum cystatin $\mathrm{C}$ levels and either anthropometric parameters,

Table 1 Comparison of anthropometric characteristics between obstructive sleep apnea patients and controls

\begin{tabular}{|c|c|c|c|c|}
\hline & Control & Patients & Test & $P$ value \\
\hline \multicolumn{5}{|l|}{ Age (years) } \\
\hline Range & $33-67$ & $40-74$ & $t=1.687$ & 0.205 \\
\hline Mean \pm SD & $49.20 \pm 9.78$ & $53.60 \pm 8.21$ & & \\
\hline \multicolumn{5}{|l|}{$\operatorname{Sex}[n(\%)]$} \\
\hline Male & $4(40)$ & $8(40)$ & $\chi^{2}=0.0$ & 1.0 \\
\hline Female & $6(60)$ & $12(60)$ & & \\
\hline \multicolumn{5}{|l|}{ Smoking $[n(\%)]$} \\
\hline Yes & $3(30)$ & $7(35)$ & $\chi^{2}=0.752$ & 0.687 \\
\hline Ex-smoker & $1(10)$ & $4(20)$ & & \\
\hline Never smoke & $6(60)$ & $9(45)$ & & \\
\hline \multicolumn{5}{|c|}{ Neck circumference $(\mathrm{cm})$} \\
\hline Range & $30-43$ & $37-49$ & $t=12.554$ & $0.001^{*}$ \\
\hline Mean \pm SD & $36.60 \pm 3.66$ & $40.95 \pm 2.91$ & & \\
\hline \multicolumn{5}{|c|}{ Hip circumference $(\mathrm{cm})$} \\
\hline Range & $100-131$ & $119-149$ & $t=35.208$ & $0.001^{*}$ \\
\hline Mean $\pm S D$ & $112.60 \pm 10.15$ & $133.15 \pm 8.31$ & & \\
\hline \multicolumn{5}{|c|}{ Waist circumference $(\mathrm{cm})$} \\
\hline Range & $97-122$ & $111-134$ & $t=28.269$ & $0.001^{*}$ \\
\hline Mean \pm SD & $107.80 \pm 9.73$ & $123.00 \pm 5.96$ & & \\
\hline \multicolumn{5}{|l|}{ Waist/hip ratio } \\
\hline Range & $0.9-0.98$ & $0.8-1.02$ & $t=4.014$ & 0.055 \\
\hline Mean \pm SD & $0.95 \pm 0.03$ & $0.92 \pm 0.05$ & & \\
\hline \multicolumn{5}{|l|}{ BMI $\left(\mathrm{kg} / \mathrm{m}^{2}\right)$} \\
\hline Range & $24.8-41.4$ & $27.3-46.3$ & $t=1.524$ & 0.227 \\
\hline Mean \pm SD & $35.1 \pm 4.6$ & $37.7 \pm 5.7$ & & \\
\hline
\end{tabular}

"Statistically significant at $P$ value less than or equal to 0.05 . 
Table 2 Statistical comparison between the two studied groups as regard different polysomnographic parameters

\begin{tabular}{|c|c|c|c|c|}
\hline & Control & Patients & $t$ test & $P$ value \\
\hline \multicolumn{5}{|l|}{$\mathrm{AHI}$} \\
\hline Range & $2-4.7$ & $7-99.3$ & 22.457 & $0.001^{*}$ \\
\hline Mean $\pm S D$ & $3.72 \pm 0.92$ & $48.94 \pm 29.90$ & & \\
\hline \multicolumn{5}{|c|}{ Average $\mathrm{SPO}_{2}(\mathrm{mmHg})$} \\
\hline Range & $95-98$ & $80-95$ & 36.901 & $0.001^{*}$ \\
\hline Mean $\pm S D$ & $96.20 \pm 1.14$ & $85.60 \pm 5.41$ & & \\
\hline \multicolumn{5}{|c|}{ Minimal $\mathrm{O}_{2}$ saturation $(\mathrm{mmHg})$} \\
\hline Range & $93-96$ & $62-92$ & 26.437 & $0.001^{*}$ \\
\hline Mean $\pm S D$ & $93.90 \pm 1.10$ & $77.45 \pm 8.80$ & & \\
\hline \multicolumn{5}{|c|}{ Baseline $\mathrm{O}_{2}$ saturation $(\mathrm{mmHg}$ ) } \\
\hline Range & $95-98$ & $93-98$ & 2.041 & 0.164 \\
\hline Mean $\pm S D$ & $96.10 \pm 0.99$ & $95.35 \pm 1.50$ & & \\
\hline \multicolumn{5}{|l|}{ TST (min) } \\
\hline Range & $245.4-372.0$ & $242.4-376.8$ & 0.000 & 0.990 \\
\hline Mean $\pm S D$ & $302.80 \pm 45.67$ & $303.0 \pm 38.79$ & & \\
\hline \multicolumn{5}{|c|}{$\mathrm{TST}<90 \%(\min )$} \\
\hline Range & $0-0$ & $3-569$ & 20.343 & $0.001^{*}$ \\
\hline Mean $\pm S D$ & $0 \pm 0$ & $209.05 \pm 145.28$ & & \\
\hline \multicolumn{5}{|c|}{$\mathrm{TST}<80 \%(\min )$} \\
\hline Range & $0-0$ & $0-372$ & 14.813 & $0.001^{*}$ \\
\hline Mean $\pm S D$ & $0 \pm 0$ & $126.10 \pm 102.70$ & & \\
\hline \multicolumn{5}{|l|}{ RDI (event/h) } \\
\hline Range & $3.4-4.7$ & $7-99.3$ & 20.581 & $0.001^{*}$ \\
\hline Mean $\pm S D$ & $4.06 \pm 0.41$ & $49.74 \pm 29.76$ & & \\
\hline \multicolumn{5}{|c|}{ Arousal index (event/h) } \\
\hline Range & $0.5-32.9$ & $0.8-118$ & 5.398 & $0.028^{*}$ \\
\hline Mean $\pm S D$ & $15.73 \pm 9.41$ & $38.94 \pm 30.63$ & & \\
\hline \multicolumn{5}{|c|}{ Snore index (event/h) } \\
\hline Range & $0.2-10.5$ & $1.5-74.3$ & 37.712 & $0.001^{*}$ \\
\hline Mean $\pm S D$ & $3.37 \pm 3.19$ & $43.03 \pm 20.12$ & & \\
\hline
\end{tabular}

$\mathrm{AHI}$, apnea-hypopnea index; RDI, respiratory disturbance index; TST less than $90 \%$, total sleep time with oxyhemoglobin saturation less than $90 \%$; TST, total sleep time; TST less than $80 \%$, total sleep time with oxyhemoglobin saturation less than $80 \%$.

* Statistically significant at $P$ value less than or equal to 0.05 .

\section{Figure 1}

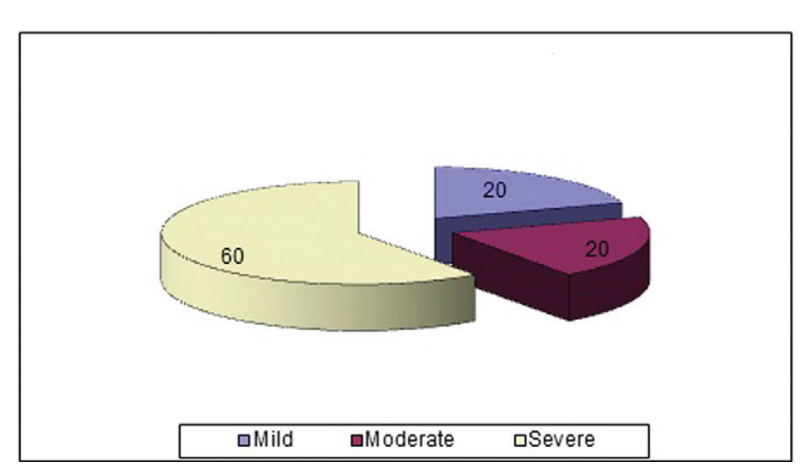

Classification of OSA severity as regard apnea-hypopnea index (AHI) showed that OSA was mild in four $(20 \%)$ patients, moderate in four $(20 \%)$ patients and severe in $12(60 \%)$ patients. OSA, obstructive sleep apnea.

age, baseline $\mathrm{O}_{2}$ saturation, serum creatinine, or eGFR by MDRD formula. A significant positive correlation was detected between serum cystatin $\mathrm{C}$ level and $\mathrm{AHI}$,
Table 3 Statistical comparison between the two studied groups regarding different laboratory investigations

\begin{tabular}{|c|c|c|c|c|}
\hline & Control & Patients & $t$ test & $P$ value \\
\hline \multicolumn{5}{|l|}{$\mathrm{pH}$} \\
\hline Range & $7.36-7.42$ & $7.35-7.44$ & 2.425 & 0.131 \\
\hline Mean \pm SD & $7.38 \pm 0.02$ & $7.40 \pm 0.03$ & & \\
\hline \multicolumn{5}{|c|}{$\mathrm{PaCO}_{2}(\mathrm{mmHg})$} \\
\hline Range & $37.1-43.2$ & $36.3-44.1$ & 0.961 & 0.335 \\
\hline Mean \pm SD & $39.27 \pm 1.89$ & $40.15 \pm 2.48$ & & \\
\hline \multicolumn{5}{|c|}{$\mathrm{PaO}_{2}(\mathrm{mmHg})$} \\
\hline Range & $80.6-101$ & $80.1-102$ & 0.499 & 0.486 \\
\hline Mean \pm SD & $87.93 \pm 5.51$ & $86.27 \pm 6.34$ & & \\
\hline \multicolumn{5}{|c|}{ Glucose (mg/dl) } \\
\hline Range & $88-112$ & $58-123$ & 0.295 & 0.591 \\
\hline Mean \pm SD & $101.30 \pm 7.86$ & $98.25 \pm 16.74$ & & \\
\hline \multicolumn{5}{|c|}{ Triglycerides (mg/dl) } \\
\hline Range & 110-239 & $107-260$ & 0.202 & 0.656 \\
\hline Mean \pm SD & $184.60 \pm 41.76$ & $177.47 \pm 40.52$ & & \\
\hline \multicolumn{5}{|c|}{ Cholesterol (mg/dl) } \\
\hline Range & $193-240$ & $129-251$ & 0.020 & 0.889 \\
\hline Mean \pm SD & $207.00 \pm 14.23$ & $205.34 \pm 35.75$ & & \\
\hline \multicolumn{5}{|l|}{ Urea (mg/dl) } \\
\hline Range & $23-37$ & $20-42$ & 1.431 & 0.242 \\
\hline Mean \pm SD & $29.00 \pm 4.81$ & $31.64 \pm 6.06$ & & \\
\hline \multicolumn{5}{|c|}{ Creatinine (mg/dl) } \\
\hline Range & $0.6-0.9$ & $0.6-1.1$ & 3.224 & 0.083 \\
\hline Mean \pm SD & $0.75 \pm 0.13$ & $0.83 \pm 0.12$ & & \\
\hline \multicolumn{5}{|c|}{ eGFR by MDRD formula $\left(\mathrm{ml} \min 1.73 \mathrm{~m}^{2}\right)$} \\
\hline Range & $65-146$ & $49-143$ & 1.764 & 0.195 \\
\hline Mean \pm SD & $102.00 \pm 32.82$ & $88.55 \pm 22.30$ & & \\
\hline \multicolumn{5}{|c|}{ eGFR by CKD-EPI equation ( $\left.\mathrm{ml} \min 1.73 \mathrm{~m}^{2}\right)$} \\
\hline Range & $80-113$ & $28-62$ & 69.933 & $0.001^{*}$ \\
\hline Mean \pm SD & $95.80 \pm 10.53$ & $50.75 \pm 8.05$ & & \\
\hline \multicolumn{5}{|c|}{ Cystatin C (mmol/l) } \\
\hline Range & $0.60-0.90$ & $0.89-2.81$ & 10.054 & $0.004^{*}$ \\
\hline Mean \pm SD & $0.73 \pm 0.11$ & $1.17 \pm 0.44$ & & \\
\hline
\end{tabular}

eGFR by CKD-EPI equation, estimated glomerular filtration rate by chronic kidney disease-Epidemiology Collaboration cystatin $\mathrm{C}$ equation; eGFR by MDRD formula, estimated glomerular filtration rate by modification of diet in renal disease; $\mathrm{PaCO}_{2}$, partial pressure of $\mathrm{CO}_{2} ; \mathrm{PaO}_{2}$, partial pressure of $\mathrm{O}_{2}$. ${ }^{*}$ Statistically significant at $P$ value less than or equal to 0.05 .

Table 4 Receiver operating characteristic curve of estimated glomerular filtration rate by chronic kidney diseaseEpidemiology Collaboration cystatin $\mathrm{C}$ equation

\begin{tabular}{lcccccc}
\hline & Cutoff & Sensitivity & Specificity & PPV & NPV & Accuracy \\
\hline eGFR by & 60 & 95 & 100 & 100 & 91 & 97 \\
CKD-EPI & & & & & & \\
equation & & & & & & \\
$(\mathrm{ml} / \mathrm{min}$ & & & & & & \\
$\left.1.73 \mathrm{~m}^{2}\right)$ & & & & & & \\
\hline
\end{tabular}

eGFR by CKD-EPI equation, estimated glomerular filtration rate by chronic kidney disease; Epidemiology Collaboration cystatin C equation; NPV, negative predictive value; PPV, positive predictive value.

TST less than $90 \%$, and TST less than $80 \%$. There was a significant negative correlation between serum cystatin $\mathrm{C}$ and average $\mathrm{SPO}_{2} \%$, minimal $\mathrm{SPO}_{2} \%$, and eGFR by CKD-EPI equation. 


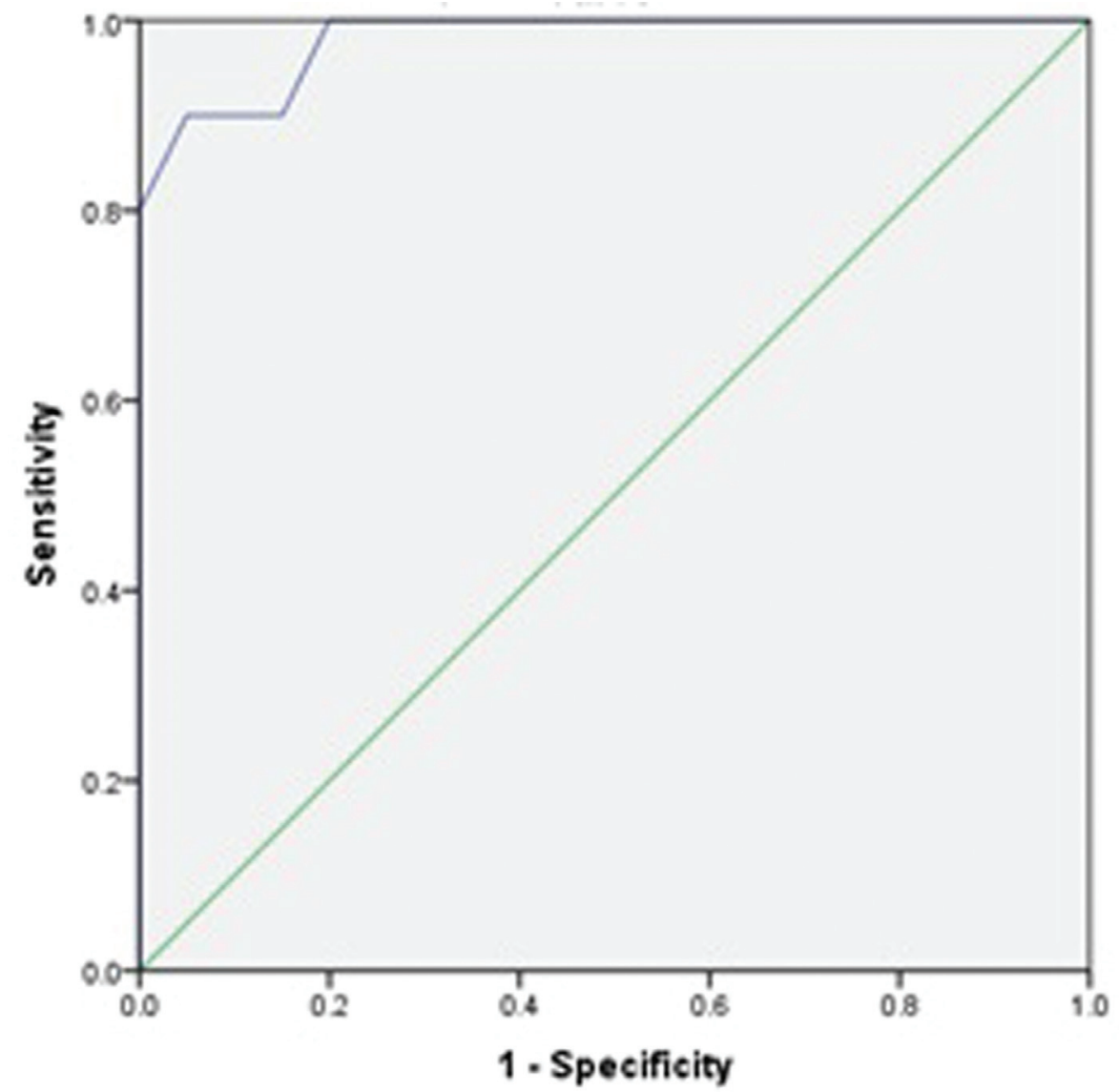

Receiver operating characteristic (ROC) curve analysis showed that cutoff value of eGFR by CKD-EPI equation in predicting renal injury was $60 \mathrm{ml} \min 1.73 \mathrm{~m}^{2}$ with a sensitivity of $95 \%$, specificity $100 \%$, PPV $100 \%$, NPV $91 \%$ and accuracy $97 \%$. CKD, chronic kidney disease; eGFR, estimated glomerular filtration rate; EPI, Epidemiology Collaboration; NPV, negative predictive value; PPV, positive predictive value.

Table 5 Statistical correlation between serum levels of cystatin $\mathrm{C}$ and some studied parameters in obstructive sleep apnea patients

\begin{tabular}{|c|c|c|}
\hline \multirow[b]{2}{*}{ Variables } & \multicolumn{2}{|c|}{ Cystatin C } \\
\hline & $r$ & $P$ \\
\hline RDI (event/h) & 0.697 & $0.022^{*}$ \\
\hline Baseline $\mathrm{O}_{2}$ saturation (\%) & -0.253 & 0.512 \\
\hline Minimal $\mathrm{O}_{2}$ saturation (\%) & -0.553 & $0.031^{*}$ \\
\hline $\mathrm{AHI}$ (event/h) & 0.786 & $0.008^{*}$ \\
\hline Age (years) & 0.131 & 0.718 \\
\hline Neck circumference $(\mathrm{cm})$ & 0.050 & 0.891 \\
\hline Hip circumference (cm) & -0.164 & 0.651 \\
\hline Waist circumference $(\mathrm{cm})$ & -0.204 & 0.572 \\
\hline Waist/hip ratio & -0.181 & 0.616 \\
\hline eGFR by MDRD formula $\left(\mathrm{ml} \min 1.73 \mathrm{~m}^{2}\right)$ & 0.424 & 0.222 \\
\hline eGFR by CKD-EPI cystatin C equation $\left(\mathrm{ml} \min 1.73 \mathrm{~m}^{2}\right)$ & -0.842 & $0.002^{*}$ \\
\hline Creatinine (mg/dl) & -0.329 & 0.354 \\
\hline TST $<90 \%$ & 0.652 & $0.024^{*}$ \\
\hline TST $<80 \%$ & 0.587 & $0.032^{*}$ \\
\hline Average $\mathrm{SPO}_{2}$ & 0.754 & $0.012^{*}$ \\
\hline
\end{tabular}

$\mathrm{AHI}$, apnea-hypopnea index; eGFR by CKD-EPI equation, estimated glomerular filtration rate by chronic kidney disease-Epidemiology Collaboration cystatin $\mathrm{C}$ equation; eGFR by MDRD formula, estimated glomerular filtration rate by Modification of diet in renal disease; $\mathrm{RDI}$, respiratory disturbance index; TST less than $90 \%$, total sleep time with oxyhemoglobin saturation less than $90 \%$; TST less than $80 \%$, total sleep time with oxyhemoglobin saturation less than $80 \%$. * Statistically significant at $P$ value less than or equal to 0.05 . 


\section{Discussion}

In this work, there was a statistically significant increase in serum cystatin C levels in OSA group than in control one. The key feature in OSA is intermittent hypoxia which appeared to have the main role in the pathological mechanism of CKD development in patients with OSA. This was proved by the significant positive correlation between cystatin C level and AHI, TST less than 90\%, TST less than $80 \%$, and RDI. However, there was a significant negative correlation between cystatin $\mathrm{C}$ and average $\mathrm{SPO}_{2} \%$, minimal $\mathrm{SPO}_{2} \%$, and eGFR by CKD$\mathrm{EPI}$ cystatin $\mathrm{C}$ equation.

In a previous study by Kato et al. [12], they found that serum cystatin $\mathrm{C}$ levels were increased in patients with severe OSA without CKD, and they considered the serum cystatin $\mathrm{C}$ as a biomarker for both clinically latent renal disease and cardiovascular risk, which is an expected complication of OSA.

Zhang et al. [13] showed in across-sectional study that serum cystatin $\mathrm{C}$ levels were relatively higher in younger men with severe OSA and in younger men without known CKD and CVD. Moreover, they found that serum levels of cystatin $\mathrm{C}$ were correlated with oxygen desaturation index, high sensitivity $\mathrm{C}$-reactive protein, serum creatinine, and eGFR. In addition, AHI was positively correlated with serum levels of cystatin C. Serum levels of cystatin C were significantly and negatively correlated with eGFR.

A study done by Archontogeorgis et al. [14] showed that higher serum levels of serum cystatin $\mathrm{C}$ were detected in middle-aged otherwise healthy patients with OSA compared with nonapneic who were matched for age and BMI, indicating that these patients are more susceptible to develop chronic kidney and CVD, and serum cystatin $\mathrm{C}$ levels were positively correlated with RDI, TST less than 90\%, and average $\mathrm{SPO}_{2} \%$ during sleep. Another study done by Chuang et al. [15] concluded that albuminuria and serum cystatin $\mathrm{C}$ levels were positively correlated with OSA severity. Moreover, Chung et al. [16], in a study conducted on 433 patients with OSA of different severity and 54 healthy controls, found that serum cystatin $\mathrm{C}$ levels in the moderate OSA group and the extremely severe OSA group were higher compared with the control group and the eGFR levels were significantly lower in the moderate and extremely severe OSA group than that in the control group.

In the present work, there was no statistically significant difference in both groups regarding eGFR by MDRD formula, whereas on comparing the mean value of eGFR by CKD-EPI cystatin C equation between the two groups, it was found to be statistically significant lower in patients with OSA than control group.

The present study depends upon CKD-EPI-cys equation but not CKD-EPIcreat-cys equation, as there is no difference in reclassification between the two equations according to KDIGO guidelines [17].

A study of Chou et al. [18] showed that high prevalence of CKD is present in patients with severe OSA without hypertension or diabetes. Significantly positive correlations were found between severity of OSA and renal function impairment.

Matsushita et al. [19] found that although CKD-EPI cystatin equation detected fewer individuals as having $\mathrm{CKD}$, it was more accurate in categorization of the risk for mortality and the development of ESRD than the MDRD study equation across a great number of participants.

Shlipak et al. [20] found that eGFR based on cystatin C alone or in combination with creatinine strengthens the association between the eGFR and the risks of death and end-stage renal disease across diverse populations more than eGFR based on creatinine.

These findings are in partial discrepancy with data from a cohort study by Rogacev et al. [21], where application of CKD-EPIcreat-cys, compared with the MDRD and CKD-EPIcreat equations, allows better prediction of CKD progression and death among patients with CKD. In a study of Zalucky et al. [22], it was found that OSA and nocturnal hypoxemia are closely related to CKD and up-regulation of the renin-angiotensin system, which is deleterious to renal function.

Doğaner et al. [23] believed that the eGFR by CKDEPI-cys $\mathrm{C}$ equation or the combined equation with creatinine has superiority to GFR equations based on creatinine alone.

Uyar et al. [24] showed that renal dysfunction seen in patients with OSA could be regarded as an additive or even synergistic result of both direct effects of disordered sleep itself and indirect effect by way of metabolic derangements.

In contrast to the recent study, Shardlow et al. [25] did not recommend the use of cystatin $\mathrm{C}$ for routine diagnosis of $\mathrm{CKD}$, and they recommended its use only in conditions where the use of serum creatinine is known to be unreliable. Moreover, they concluded that the use of 
cystatin $\mathrm{C}$ did not improve the prediction of the risk for mortality or deterioration of CKD. The difference between their observation and ours can be explained by the differences in the aim of both studies, as they used cystatin $\mathrm{C}$ as a marker for detection of the severity of CKD in already diagnosed cases, but in the present study, the aim was to use cystatin $\mathrm{C}$ in early detection of renal impairment in apparently healthy individuals, in addition to the big difference in the number of targeted populations in both studies.

In the present study, eGFR by MDRD formula in all participants was not significantly affected because it depends mainly on creatinine level, which was not also significantly affected, as participants had not yet developed CKD, in contrast to eGFR by CKD-EPI cystatin equation, where it was statistically significant lower in patients with OSA than control group, as it depends mainly on cystatin $\mathrm{C}$ levels, which were statistically significant higher in patients with OSA, and it is a predictor of early and latent renal dysfunction.

Our study had several limitations: limited number of the study population and limited time of the study. Moreover, it is a case-controlled study, so follow-up is needed to determine the probable future prevalence of chronic renal disease in patients with OSA with high serum cyst $\mathrm{C}$ levels. Moreover, there is a probability that this study included patients having CKD despite their normal eGFR because proteins and albumin in urine had not been measured. Most of participants who were included in this analysis were elderly and had high prevalence of major chronic diseases, which were associated with a higher risk of CKD.

In conclusion, detection of higher levels of serum cystatin $\mathrm{C}$ in otherwise healthy patients with OSA could indicate an increased future risk of developing $\mathrm{CKD}$ in these patients. Intermittent hypoxia seems to play a central role in the progression of this process.

\section{Financial support and sponsorship}

Nil.

\section{Conflicts of interest}

There are no conflicts of interest.

\section{References}

1 Banno K, Manfreda J, Walld R, Walld R, Delaive K, Kryger M. Healthcare utilization in women with obstructive sleep apnea syndrome 2 years after diagnosis and treatment. Sleep 2006; 29:1307-1311.

2 Fleischmann G, Fillafer G, Matterer H, Skrabal F, Kotanko P. Prevalence of chronic kidney disease in patients with suspected sleep apnoea. Transplant 2010; 25:181-186.
3 Nicholl DDM, Ahmed SB, Loewen AHS, Hemmelgarn BR, Sola DY, Beecroft JM, et al. Declining kidney function increases the prevalence of sleep apnea and nocturnal hypoxia. Chest 2012; 141;1422-1430.

4 Blaydon DC, Nitoiu D, Eckl KM, Cabra RM, Bland P, Hausser I, et al. Mutations in CSTA, encoding cystatin A, underlie exfoliative ichthyosis and reveal a role for this protease inhibitor in cell-cell adhesion. Am J Human Genet 2011; 89;564-571.

5 Dharnidharka VR, Kwon C, Stevens G. Serum cystatin C is superior to serum creatinine as a marker of kidney function: a meta-analysis. $A m \mathrm{~J}$ Kidney Dis 2002 40;221-226.

6 American Academy of Sleep Medicine. International classification of sleep disorders. 3rd ed. Darien, IL: American Academy of Sleep Medicine; 2014.

7 Johns MW. A new method for measuring daytime sleepiness: The Epworth Sleepiness Scale. Sleep 1991; 14:540-545.

8 Berry RB, Brooks R, Gamaldo CE, Harding SM, Lloyd RM, Marcus CL, Vaughn BV, for the American Academy of Sleep Medicine. The AASM manual for the scoring of sleep and associated events: rules, terminology and technical specifications, Version 2.3: American Academy of Sleep Medicine, Westchester, IL, 2016.

9 The Expert Committee on the Diagnosis and Classification of Diabetes Mellitus. Follow-up report on the diagnosis of diabetes mellitus. Diabetes Care 2003; 26:3160-3167.

10 Levey AS, Stevens LA, Schmid CH, Zhang YL, Castro AF, Feldman HI, et al. A new equation to estimate glomerular filtration rate. Ann Intern Med 2009; 150;604-612.

11 Levey AS, Stevens LA. Estimating GFR using the CKD Epidemiology Collaboration (CKD-EPI) creatinine equation: more accurate GFR estimates, lower CKD prevalence estimates, and better risk predictions. Am $J$ Kidney Dis 2010; 55:622-627.

12 Kato K, Takata Y, Usuietal Y. Severe obstructive sleep apnea increases cystatin C in clinically latent renal dysfunction. Respir Med 2011; 105:643-649.

13 Zhang XB, Lin QC, Deng CS, Chen GP, Cai ZM, Chen H, et al. Elevated serum cystatin $C$ in severe OSA younger men without complications. Sleep Breath 2013; 17:235-241.

14 Archontogeorgis K, Nena E, Tsigalou C, Voulgaris A, Xanthoudaki M, Froudarakis $\mathrm{M}$, et al. Cystatin $\mathrm{C}$ levels in middle-aged patients with obstructive sleep apnea syndrome. Pulm Med 2016; 2016:8081723.

15 Chuang LP, Lin SW, Lee LA, Chang $\mathrm{CH}$, Huang HY, Chung $\mathrm{H}$. Elevated serum markers of acute kidney injury in patients with obstructive sleep apnea. J Clin Sleep Med 2019; 15:207-213.

16 Chung L, Yan EB, You H, Wai J, Zhi Z. Preliminary study on the relationship between OSA and renal function. Lin Chung Er Bi Yan Hou Tou Jing Wai Ke Za Zhi 2019; 33:298-303.

17 Beben T, Rifkin DE. GFR estimating equations and liver disease. Adv Chronic Kidney Dise 2015; 22:337-342.

18 Chou YT, Lee PH, Yang CT, Lin CL, Veasey S, Chuang LP, et al. Obstructive sleep apnea: a stand-alone risk factor for chronic kidney disease. Nephrol Dialy Transplant 2011; 26:2244-2250.

19 Matsushita K, Mahmoodi BK, Woodward M, Emberson JR, Jafar TH, Jee $\mathrm{SH}$, et al. Chronic Kidney Disease Prognosis Consortium. Comparison of risk prediction using the CKD-EPI equation and the MDRD study equation for estimated glomerular filtration rate. JAMA 2012; 307:1941-1951.

20 Shlipak MG, Matsushita K, Ärnlöv J, Inker LA, Phil RK, Polkinghorne KR, et al. Cystatin $\mathrm{C}$ versus creatinine in determining risk based on kidney function. New Engl J Med 2013; 369:932-943.

21 Rogacev KS, Pickering JW, Seiler S, Zawada AM, Emrich I, Fliser D, et al The Chronic Kidney Disease Epidemiology Collaboration (CKD-EPI) equation incorporating both cystatin $\mathrm{C}$ and creatinine best predicts individual risk. Nephrol Dial Transplant 2014; 29:348-355.

22 Zalucky AA, Nicholl DD, Hanly PJ, Poulin MJ, Turin TC, Walji S, et al. Nocturnal hypoxemia severity and renin-angiotensin system activity in obstructive sleep apnea. Am J Respir Crit Care Med 2015; 192;873-880.

23 Doğaner YÇ, Aydoğan Ü, Rohrer JE, Aydoğdu A, Çaycı T, Barçın C, et al. Comparison of estimated GFR equations based on serum cystatin $C$ alone and in combination with serum creatinine in patients with coronary artery disease. Anatol J Cardiol 2015; 15:571.

24 Uyar M, Davutoğlu V, Gündoğdu N, Kosovalı D, Sarı i. Renal functions in obstructive sleep apnea patients. Sleep Breath 2016; 20:191-195.

25 Shardlow A, McIntyre NJ, Fraser SD, Roderick P, Raftery J, Fluck RJ, et al. The clinical utility and cost impact of cystatin $C$ measurement in the diagnosis and management of chronic kidney disease: a primary care cohort study. PLoS Med 2017; 14:e1002400. 